Michel, GF. A holistic developmental theory requires better research techniques. Behavioral \& Brain Sciences. 1999, 22(5): 899-900.

Made available courtesy of Cambridge University Press: http://journals.cambridge.org/action/login

\title{
A holistic developmental theory requires better research techniques
}

\author{
George F. Michel
}

Psychology Department, DePaul University, Chicago, IL 60614-3504.

gmichel@condor.depaul.edu depaul.edu/ gmichel

\begin{abstract}
Research pragmatics, not a defective conceptual framework, supports modern biological reductionism. Conducting research to reveal the casual web underlying the multiple developmental pathways leading to any species-specific characteristic requires better research techniques than those commonly used. It takes much patience, time, and effort to gain even small glimpses of an answer to any developmental question.

Lifelines presents a coherent account of living systems that integrates physics, chemistry, and biology in a way that is intended to undercut any attempt to use only reductionistic explanations of the human condition. As Rose notes, deterministic biological explanations resurfaced during the late 1960s without any specific advance in biological science or theory. However, they have been advanced (to the dismay of thoughtful scientists) by recent biomedical and popular interpretations of modern molecular genetics and neuroscience. Rose argues that the reductionist trend stems from both a tradition of determinism in biological training and thinking and the failure of "opponents of biological determinism ... to offer a coherent alternative framework within which to interpret living processes" (p. ix). However, many such frameworks have been proposed during the last 100 years and several quite recently (cf. Elman et al. 1996; Gottlieb et al. 1998; Michel \& Moore 1995). Why then does reductionism persist?

The reductionist tradition certainly influences the way many biologically oriented investigators pose research questions, apply re-
\end{abstract}


search techniques, interpret findings, and train students. It does so because this is more efficient in generating research results and providing ready interpretations than research that "celebrates complexity" and focuses on the specific dynamics of the organism- environment system over its life-span. While I appreciate Rose's efforts, biological determinism will continue to dominate because unraveling the organismic-experiential causal web underlying the multiple developmental pathways of species-typical characteristics is not easily done, nor is there a generally accepted set of techniques for doing so.

In contrast, researchers in neuroscience, molecular and behavior genetics, sociobiology, and cognitive science, have shown that it is relatively easy to conduct research on questions of proximate causation, phylogenetic relationships, and adaptive function. There are many ways to investigate the contributions of genes and various neural processes and structures to behavior and many of these involve exciting new technologies to attract popular interest. Of course, only reductionism assumes the nervous system to be organized by genecontrolled processes (Rose nicely illuminates the difference between genetic involvement and genetic control). Sociobiology has demonstrated the efficiency of collecting data that address alternative game-theoretical or economic models of generalized types of behavior, presumably controlled by genes. Such results fit current thinking about adaptation and natural selection so well that they tempt researchers away from pursuing developmental questions.

In contrast, typical developmental research techniques are limited and problematic. Many studies involve correlating individual differences earlier and later in development. This does not reveal whether the individuals have changed between measurement periods (a developmental event). Nor do these studies reveal the developmental processes that contribute to maintaining relative position across age periods. The absence of correlation may be interpreted by reductionists as indicating that early developmental events have no impact on later events because they are biologically controlled. Alternatively, high correlation may be interpreted as strong biological control during that age period.

Other developmental techniques examine the relation between a manipulation (or clinical event) occurring earlier in development and the individual's characteristics later in development. Even if a relation exists, nothing is revealed about the causal web connecting the two developmental periods. If a relation does not exist, nothing is revealed about the processes that blocked the connection.

A common technique of depriving an organism of certain experiences to determine their influence on development presumes that one already knows or suspects that such experiences contribute to development. A few carefully crafted programmatic studies have revealed that development can depend upon experiences which do not at first appear to be relevant. Thus, the absence of deprivation effects only means that the missing experiences were not relevant for that developmental outcome, or if they were, that their loss could be compensated for by other means. For example, in some bird species, song can develop without hearing other singing birds or the bird's own singing, but that does not rule out other auditory experience. Even if for some species all auditory experience were ruled out, other experiences (e.g., vestibular and/or respiratory experience, or specific social experiences) could be involved (Clayton 1994). Thus, although deprivation studies can fail to reveal how develop occurs, they can support reductionist interpretations.

Since models of learning often fail to provide insight into the development of many species-typical characteristics, some argue that individual experience is unimportant for them. However, learning models represent only a small part of the range of experiential events that contribute to development, some of them seemingly irrelevant. Most stimulation studies fail to identify the experiential influences on development because they provide either a broad-band level of stimulation that approximates those that are characteristic of the natural environment more than the usual laboratory situation, or they provide unusually high levels of very complex stimulation. No one would deny that approximating a normal environment can support normal development or that normal development can be disrupted by stress or overstimulation. Hence such studies do not challenge reductionism. Only a few studies (e.g., bird-song, imprinting, sex differences in rat behavior) have manipulated levels and patterns of stimulation within the bounds of their normal occurrence to identify their contribution to the development of species-typical behavior (cf. Gottlieb et al. 1998; Ten Cate 1994; Moore 1992). In each case, such experiences are profoundly involved in the normal development of the species-typical behavior. [See also Baker: "The Biology of Bird-Song Dialects" BBS 8(1) 1985; Johnston: "Development, Explanation and the Ontogeny of Bird Song-Nature" BBS 11(4) 1988.]

Careful, time-consuming, programmatic investigations need to be encouraged if we are to understand development and avoid what some see as the pitfalls of reductionism. 